\title{
Tibetische Rezepturen als pleiotrope Signaturen - Einsatz von Netzwerk-Arzneien bei Multimorbidität
}

\author{
Herbert Schwabl $^{\mathrm{a}} \quad$ Cécile Vennos $^{\mathrm{a}}$ Reinhard Saller \\ a Padma AG, Hinwil, \\ b Institut für Naturheilkunde, Universitätsspital Zürich, Schweiz
}

\section{Schlüsselwörter}

Netzwerk-Arzneien - Netzwerk-Pharmakologie · Pleiotropie . Systembiologie · Multimorbidität · Multi-Target-Mechanismus . Multitherapeutic · Tibetische Medizin · Phytotherapie

\section{Zusammenfassung}

Multimorbide Zustände ( $\geq 2$ chronische Erkrankungen gleichzeitig) können vor dem Hintergrund des Netzwerkmodells des Organismus als komplexe Krankheitsmuster aufgefasst und als charakteristische Signaturen abgebildet werden. Lebende Systeme wie der menschliche Organismus werden aus der Sicht der Systemtheorie als Netzwerke von interagierenden Teilen betrachtet. Diese können wiederum selbst Unternetzwerke sein und verschiedenen Komplexitätsstufen zugeordnet werden. Sie reichen z.B. vom Gen über das Transkriptom, Proteom, Metabolom und Epigenom bis hin zum Netzwerk der gesamten molekularen Interaktionen, dem sogenannten Interaktom. Bei der Multimorbidität betrifft die Krankheitssignatur verschiedene Netzwerke auf allen Stufen, z.B. Zellsysteme, Organe und funktionelle Systeme. Basierend auf dieser Semiotik können auch Arzneimitteln bestimmte Wirksignaturen bzw. Wirkprofile zugeordnet werden. Eine Pharmakonsignatur repräsentiert die physikalisch-chemischen Reize, die das System zu einer Reaktion veranlassen, sowie die Verbindungen, über die das vernetzte Gesamtsystem auf allen Hierarchiestufen beeinflusst wird. Phytotherapeutika, die chemisch gesehen Vielstoffgemische darstellen, besitzen besonders komplexe Signaturen. Als Multi-Target-Arzneien mit pleiotropem Wirkprofil greifen sie auf den unterschiedlichen Systemstufen des Netzwerks therapeutisch ein, weshalb sie auch als Netzwerk-Arzneien bezeichnet werden. Als Beispiel für Phytotherapeutika mit besonders komplexer pleiotroper Signatur können Pflanzenrezepturen traditioneller Medizinsysteme wie der Tibetischen Medizin herangezogen werden. Hier wird auch aus traditioneller Sicht eine Krankheitssignatur mit einer korrespondierenden Arzneimittelsignatur in Beziehung gesetzt, jedoch basierend auf dem traditionellen energetischen Verständnis von Krankheiten. Moderne Forschungsergebnisse geben deutliche Hinweise auf eine ausgesprochen breit gefächerte Signatur Tibetischer Arzneimittel und liefern somit die logische Grundlage für ihren Einsatz im Rahmen integrativer Behandlungsansätze bei Krankheiten mit ebenfalls komplexer Signatur, z.B. bei Multimorbidität. Der vorgestellte systemtheoretische Ansatz stellt eine Methode dar, die Anschlussfähigkeit traditioneller und komplementärmedizinischer Methoden an die anderen Disziplinen der modernen Medizin zu ermöglichen.

\section{Keywords \\ Network drugs · Network pharmacology · Pleiotropy · Systems biology · Multimorbidity · Multi-target mechanisms . Multi-therapeutic drugs · Tibetan Medicine · Phytotherapy}

\section{Summary}

Tibetan Formulas as Pleiotropic Signatures -

Application of Network Medicines in Multimorbidity

In the context of the network model of the organism, multimorbid states ( $\geq 2$ chronic diseases at the same time) can be considered as a complex disease pattern which can be mapped as characteristic signatures. From the perspective of system theory, living systems such as the human body are viewed as networks of interacting parts. These in turn can themselves be subnetworks assigned to different complexity levels. They range, e.g., from the gene to the transcriptome, proteome, metabolome, and epigenome up to the network of the entire molecular interactions, the so-called interactome. In multimorbidity, the disease signature affects different networks at all levels, e.g., cell systems, organs, and functional systems. Based on this semiotics, certain signatures of effectiveness and profiles of action can be assigned to each drug. A drug signature represents the physicochemical stimuli that cause a reaction by the system, as well as the cross-links by which the entire connected system is affected at all levels. Phytotherapeutics, which chemically represent multi-component mixtures, have especially complex signatures. As multi-target medicines with a pleiotropic effect profile, they therapeutically affect different levels of the network, which is why they are also referred to as network medicines. Herbal formulas from traditional medicine systems such as Tibetan Medicine are an example for phytotherapeutics with a particularly complex pleiotropic signature. Also from the traditional point of view, a disease signature is set in relation with a corresponding drug signature. However, in this case, it is based on the traditional energetic understanding of diseases. Modern research results clearly indicate a widely diversified signature range of Tibetan Medicines and thus provide a rationale for their use in integrative treatment approaches for diseases with complex signatures, e.g. in multimorbidity. The system-theoretical approach discussed here represents a method to enable a connectivity of traditional methods from complementary and alternative medicine to the other disciplines of modern medicine.

\section{KARGER \\ Fax +497614520714 \\ Information@Karger.com}

www.karger.com (c) 2013 S. Karger GmbH, Freiburg

$1661-4119 / 13 / 0208-0035 \$ 38.00 / 0$

Accessible online at:

www.karger.com/fok
Dr. med. vet. Cecile Vennos

Padma AG

Unterfeldstrasse 1,8340 Hinwil, Schweiz

c.vennos@padma.ch 


\section{Multimorbidität als Herausforderung}

Multimorbidität wird als das gleichzeitige Vorhandensein von 2 oder mehr chronischen Krankheiten oder Zuständen («conditions») definiert, wobei keine mehr im Zentrum der Betrachtung steht als die anderen. Demgegenüber steht bei Komorbidität definitionsgemäß eine Indexerkrankung im Vordergrund.

In allen modernen Gesellschaften steigt, nicht zuletzt aufgrund der höheren Lebenserwartung im Rahmen des medizinischen Fortschritts, die Anzahl der chronisch Kranken. Dabei nimmt auch die Multimorbidität mit gleichzeitigem Vorliegen mehrerer chronischer Erkrankungen zu. Sie hat sich weltweit, und so auch in der Schweiz, bei Erwachsenen zu einer der individuell, sozialmedizinisch und ökonomisch bedeutendsten Krankheitssituationen entwickelt [1]. Bei der Erhebung von Multimorbidität ist das Spektrum der jeweils miteinbezogenen Erkrankungen sehr unterschiedlich. Es reicht von wenigen Krankheiten (5-10) bis zu >300 Diagnosen (entsprechend der International Classification of Diseases 10 (ICD-10)). Die Aufschlüsselung der berücksichtigten Erkrankungen ergibt ein sehr heterogenes Bild. Besonders uneinheitlich erweist sich die Handhabung bei kardiovaskulären Erkrankungen (als Gesamtgruppe oder nach ausgewählten Einzelerkrankungen wie Hypertonie, Herzinsuffizienz, Schlaganfall) und insbesondere der Einbezug bzw. Nichteinbezug psychischer Erkrankungen.

Nicht zuletzt in der Behandlungspraxis zeigt sich, dass Multimorbidität etwas anderes zu sein scheint als nur die Summe der erfassten Einzelerkrankungen. Über die Aufsummierung hinaus stellt sie ein zumindest partiell eigenständiges, vielgestaltiges und dynamisches chronisches Krankheitsbild dar. Die dazu beitragenden Erkrankungen können konkordant sein, wie etwa Hypertonie, koronare Herzkrankheit und Diabetes, oder diskordant, wie z.B. Herz-Kreislauf-Erkrankungen als Gruppe zusammengefasst gemeinsam mit chronisch obstruktiver Lungenerkrankung oder Arthritiden. Vielfach sind auch seelisch-körperliche Zusammenhänge feststellbar, vor allem das gleichzeitige Auftreten depressiver Zustände. Mit steigen- der Anzahl physischer chronischer Krankheitszustände tragen Patienten ein zunehmendes Risiko für psychische Erkrankungen, vor allem Depressionen. Umgekehrt haben Patienten mit seelischen Erkrankungen ein hohes Risiko für die Entwicklung chronischer somatischer Erkrankungen [2].

In der modernen Pharmakotherapie ist die Antwort auf das Phänomen der Multimorbidität oft unbefriedigend oder mangelhaft. Hochspezifische Wirkstoffe allein, die an einzelne Liganden binden («Single Targets»), können diesem Thema nicht gerecht werden. Es kommt typischerweise zu Polypharmakotherapien, womit auch das Thema der Arzneimittelinteraktionen an Bedeutung zunimmt.

\section{Multimorbidität als Signatur komplexer Systeme}

Aus der Sicht der Systemtheorie kann die Multimorbidität als eine typische Signatur des komplexen Systems «Organismus» gesehen werden. Verbunden mit der Grundkonstitution (genetisch, epigenetisch, Umwelt, Lebensweise, Alter, Geschlecht) bildet sie die Antwort auf die vielfältigen äußeren Einflüsse $a b$.

Lebende Systeme, so auch der menschliche Organismus, werden systemtheoretisch als Netzwerke von interagierenden Teilen betrachtet, die verschiedene Informations- und Regelkreise umfassen. Das oberste Ziel eines solchen autonomen Systems ist dabei die Homöostase, d.h. die Integrität des Systems unter verschiedenen äußeren und inneren Einflüssen durch Autopoiese intakt zu erhalten [3, 4].

Die Netzwerkelemente im Organismus [5, 6] bestehen wiederum aus eigenständigen Subnetzwerken, was man als Hierarchie der Netzwerke beschreiben kann. Die unterschiedlichen Systemstufen unterscheiden sich dabei nach innerer Bindungsstärke, bezüglich der charakteristischen Reaktionszeit sowie in der Art und Anzahl der Signale, die verarbeitet werden können (Abb. 1). Je komplexer ein System, umso gröBer ist sein Repertoire an möglichen verarbeitbaren bzw. beantwortbaren Signalen. Dieses Interaktionspotenzial definiert
Abb. 1. Schema der Netzwerk-Hierarchie im menschlichen Organismus. Jede Hierarchiestufe kann durch Umweltfaktoren beeinflusst werden (----). Rot hervorgehoben ist eine beispielhafte Signatur, die die verschiedenen angesprochenen Signalwege umfasst. Die Dicke der Linien kennzeichnet die Verbindungsstärke. Dabei ist die Signatur phytotherapeutischer Vielstoffgemische charakteristischerweise deutlich komplexer als bei Single-Target-Arzneimitteln.

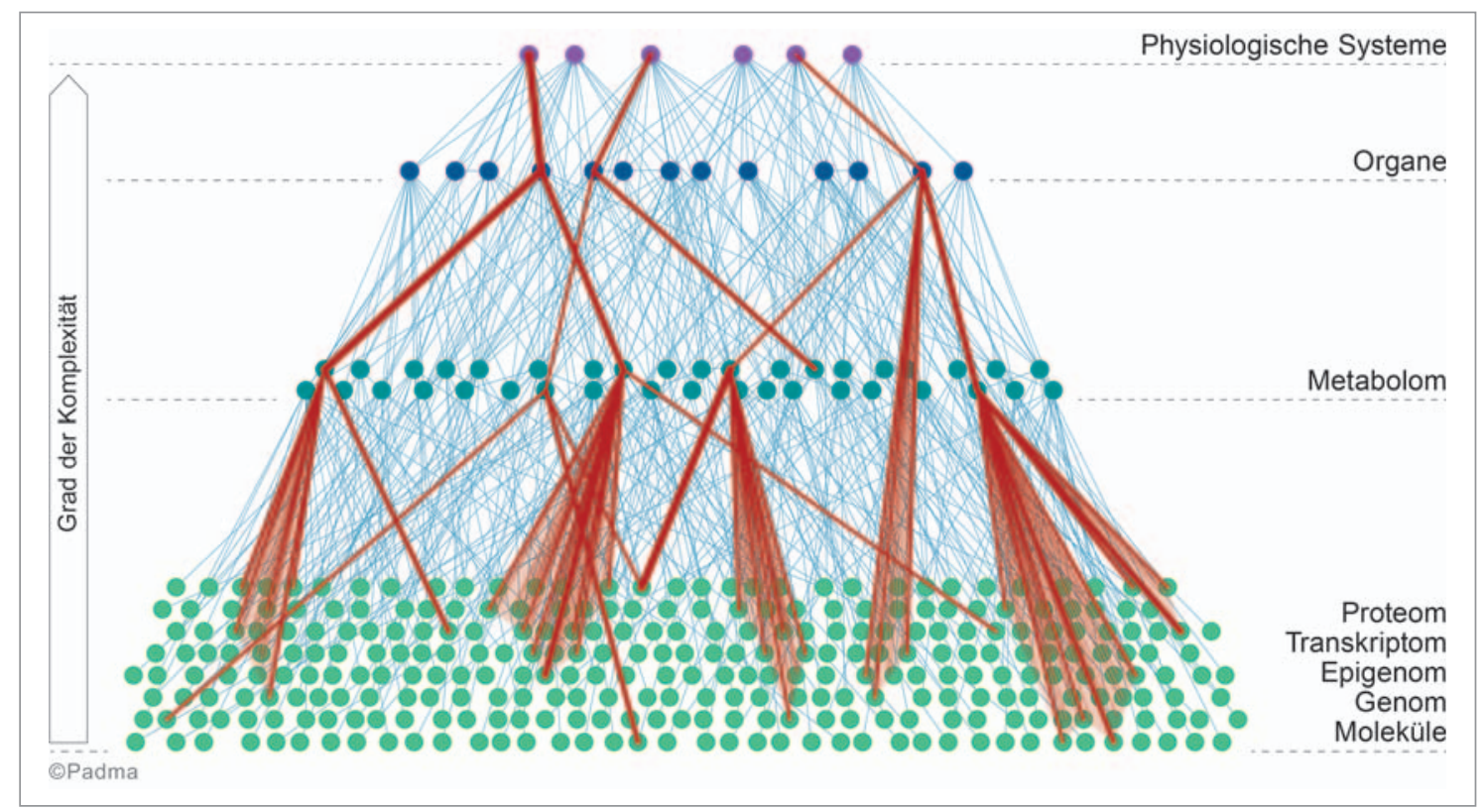

Forsch Komplementmed 2013;20:35-40
Schwabl/Vennos/Saller 
die charakteristische Semiotik jedes Systems als Basis der möglichen Interaktionen mit dem System sowie das mögliche Antwortrepertoire. Bei der Wahl einer therapeutischen Intervention und der Planung eines Behandlungsprotokolls müssen die Art der therapeutischen Signale (manuell, invasiv, pharmakologisch, narrativ) sowie deren Abfolge der semiotischen Kapazität des Systems entsprechen.

Auch Krankheiten betreffen nicht einen einfachen linearen Pfad, sondern das gesamte Netzwerk [7]. Zum Beispiel wird die Entstehung und der Krankheitsverlauf der Atherosklerose neben vielen bekannten exogenen Risikofaktoren auch durch Netzwerkelemente des Organismus auf verschiedenen Ebenen beeinflusst: durch die Grundkonstitution auf der Basis des Genoms, durch das Metabolom mit den verschiedenen Zelltypen, Zellstoffwechseln und Organsystemen [8] sowie durch das damit zusammenhängende Epigenom und seine Beeinflussung durch die Lebensweise. Entsprechend vielfältig sind die diagnostischen und therapeutischen $\mathrm{Zu}$ gänge. Einerseits umfassen sie physiologische Systeme und Organe wie das Herz-Kreislauf-System, Bluthochdruck, Koagulation, Diabetes, Plasmalipide oder Adipositas. Andererseits geht es auf der zellulären Ebene um Themen wie chronische Entzündung, Hormonstoffwechsel, Endothelfunktion oder Fettgewebe. Diese exogenen und endogenen Aspekte der multimorbiden Krankheitssignatur sind auf die reaktive Kapazität des Netzwerks Organismus abzustimmen und in ein individualisiertes Therapieprofil mit einzubeziehen.

\section{Vom Netzwerk zur Signatur}

Komplexe Netzwerke haben eine Reihe von gemeinsamen Charakteristika [6]:

- Kleine-Welt-Struktur («small-worldness»): Es bestehen viele Abkürzungswege, die die verschiedenen Netzwerkelemente direkt miteinander verbinden. Dies führt oft zu scheinbar paradoxalen (nichtlinearen) Ergebnissen bei der Interaktion mit einem komplexen System. Eine lokale Applikation kann sich so rasch und disloziert auf das ganze System auswirken, was sehr gut anhand der Effekte der Akupunktur gezeigt werden kann.

- Skalenfreiheit («scale-free topology»): Es gibt verteilte Netzwerkknoten, die eine wesentlich höhere Anzahl von Verbindungen haben als die benachbarten Knoten. Im Rahmen eines Therapiekonzepts bei Multimorbidität scheint es zielführend, hoch verbundene Netzwerkknoten $\mathrm{zu}$ finden und therapeutisch zu beeinflussen.

- Die Netzwerke sind in überlappende Gruppen aufgeteilt. Es gibt keine scharfen Grenzen zwischen den einzelnen Netzwerken, dies ist auch eine Konsequenz des redundanten Aufbaus unseres Organismus als komplexes System. Eine streng binär aufgebaute Beschreibung kann das System daher nicht vollständig erfassen.

- Koexistenz von starken und schwachen Verbindungen. Als stark wird eine Verbindung gewertet, wenn sie starke physikalisch-chemische Bindungskräfte mit hoher Ansprechwahrscheinlichkeit beinhaltet. Zusätzlich kann die Stärke jeder Verbindung und damit des ganzen Verbindungsmusters zeitlich variieren.
Aufgrund dieser Eigenschaften kann zum besseren Verständnis ein vereinfachtes Netzwerkmodell konstruiert werden, das die wesentlichen Pfade innerhalb des Netzwerks umfasst und auf dem sich für eine Krankheit oder einen Krankheitskomplex ein Muster bzw. eine Signatur abbilden lässt. Der heute aktuelle Forschungsansatz der Systembiologie bezieht Systeme verschiedener Hierarchiestufen mit ein, von der Gen-Transkript-Stufe über das Proteom, Metabolom und Epigenom hin zum Netzwerk der gesamten molekularen Interaktionen, dem sogenannten Interaktom $[5,9]$.

Überträgt man diese Sicht auf die Pharmakologie, gilt als Signal jeweils ein physikalisch-chemischer Reiz, der zumindest ein Element eines Subsystems zu einer Reaktion veranlasst. Über die vernetzten Hierarchiestufen wird jedoch auch das Gesamtsystem beeinflusst. Damit kann man auch von einer Signatur eines Pharmakons sprechen, die das Reaktionsbild über die verschiedenen Hierarchiestufen hinweg abbildet (Abb. 1). Aus systembiologischer Sicht bedeutet das, dass der bislang in der Pharmakologie favorisierte Ansatz der «magic bullet», d.h. einen spezifischen Liganden («single target») anzusprechen, zu simplifizierend ist [6]. Im Gegensatz dazu treten sogenannte Multi-Target-Arzneien ins Zentrum des Interesses. Diese greifen in sinnvoller Dosierung an mehreren Wirkorten ein, allerdings ohne z.B. diese Targets vollständig zu hemmen (partielle Inhibition). Solche partiellen Hemmungen können wirksamer sein als die vollständige Hemmung eines Single-Targets [10] und sie scheinen zudem verträglicher zu sein. Analog zu den Auswirkungen der Multimorbidität auf den Kranken über verschiedene Netzwerkhierarchien wirken solche Multi-Target-Arzneien ebenfalls über Netzwerkhierarchien auf das Gesamtsystem.

\section{Pflanzliche Arzneimittel: Von Multi-Target Drugs zu Netzwerk-Arzneien}

Phytotherapeutika sind chemisch gesehen Vielstoffgemische und damit typische Multi-Target-Arzneien (Abb. 2) [1113]. Die Wirkstoffe lassen sich mit folgenden Eigenschaften beschreiben [11-15]:

- zahlreiche aktive Prinzipien, niedrig konzentrierte Einzelkomponenten;

- die einzelnen Wirkstoffbestandteile liegen meist nur in sehr geringen Mengen vor, dadurch keine vollständigen Blockaden/Stimulierungen/Sättigungen;

- multiple, pleiotrope (d.h. voneinander unabhängige) Mechanismen und dadurch gleichzeitige Ansprache mehrerer Mechanismen im Netzwerk;

- schwache Bindungen;

- ausgeprägte Plastizität des Wirkstoffs, d.h. seine dynamische Komplexität kann sich der dynamischen Multimodalität der Targets anpassen, noch dazu mit relativ wenigen Nebenwirkungen.

Signaturen und Potenziale phytotherapeutischer Wirkstoffe sind in Tabelle 1 zusammengefasst.

Entsprechend dem breiten Wirkungsspektrum kommt es bei der phytotherapeutischen Arzneimittelwahl aufgrund der Multifunktionalität von Phytotherapeutika [16] meistens zu einer Überschneidung der 3 Wirkebenen: 
Tab. 1. Allgemeine Charakteristika bzw. Signatur phytotherapeutischer Wirkstoffe sowie ihre Anwendungseigenschaften und -möglichkeiten
Wirkstoffcharakteristika

Genuines Vielstoffgemisch

Allgemeine Betrachtung

Strukturelle Betrachtung

Funktionelle Betrachtung

Pleiotrope Eigenschaften

Multi-Target-Eigenschaften

Therapeutische Anwendung

Multitherapeutic-Eigenschaften

Indikationsbezogen

Als Systemmittel

Komplexe Krankheitsbilder bzw. Therapiesituationen, z.B. Multimorbidität
Vielstoffgemisch in Einzeldrogen bzw. Einzelextrakten sowie in Drogen- und Extraktkombinationen

Ansammlung vieler Bestandteile (Wirkstoffkomponenten in meist sehr niedrigen Konzentrationen)

Wirkungsgefüge aufgrund vielfältiger Effekte und drogen- bzw. organismusbezogener Interaktionsmöglichkeiten (Netzwerkmodell, Plastizität)

gleichzeitig mehrere bis zahlreiche voneinander unabhängige Wirkmechanismen (polypharmakologischer Ansatz)

multiple, multifokale und multimodale Wirkungen; Multi-Target-Eigenschaften auf molekularer und zellulärer Ebene sowie krankheitsbezogen und im Hinblick auf eventuelle Präventionsansätze (polypharmakologischer Ansatz)

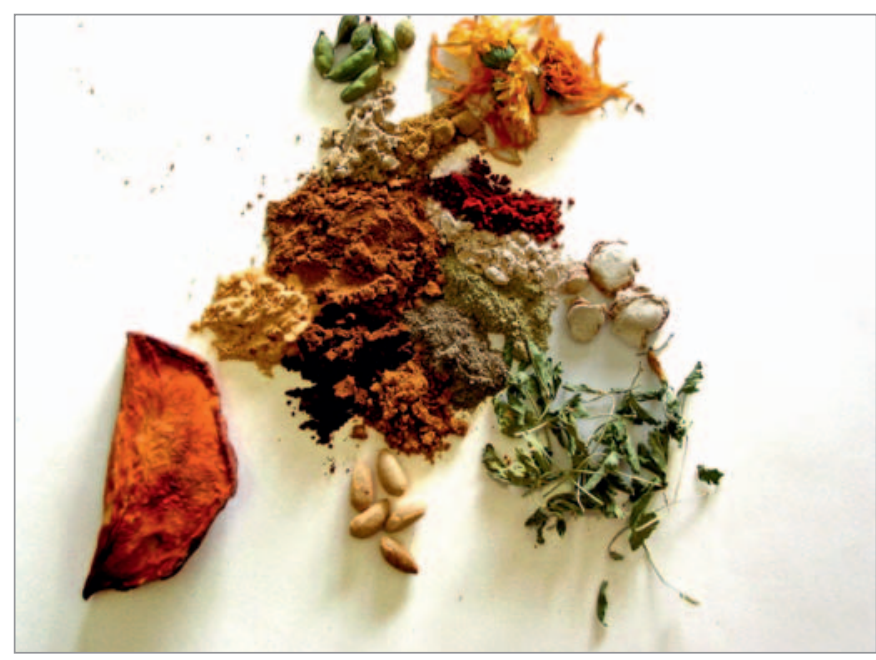

Abb. 2. Tibetische Arzneimittel sind Vielstoffgemische. Im Beispiel einige Inhaltsstoffe der Rezeptur Padma 28.

- spezifisch: Orientierung an Symptomen/Symptomkomplexen bzw. spezifischen Krankheiten;

- adaptogen: Orientierung am Gesamtorganismus und seinen Reaktionsweisen;

- systemisch: Orientierung an Basisstörungen (z.B. Entzündungsvorgänge).

Überträgt man diese Gedanken auf das Netzwerk, so wird schnell erkennbar, dass jedem Phytotherapeutikum eine charakteristische Signatur zukommt, weshalb sie auch als NetzwerkArzneien [17] bezeichnet werden (Abb. 3). Durch den pleiotropen Charakter ist dieses Muster breiter als bei Monosubstanzen und auf viele, vor allem schwach ausgeprägte
Verbindungen ausgelegt [18]. Insbesondere bei pflanzlichen Vielkomponenten-Rezepturen, die durch die erfahrungsmedizinische Erprobung entwickelt wurden, sind das Multi-TargetKonzept und verbunden damit die Pleiotropie stark ausgeprägt. Solche Kombinationspräparate können sich besonders als eine systemische Basistherapie eignen, z.B. kombiniert mit selektiven Arzneimitteln zur Behandlung multimorbider Patienten.

\section{Empirisch erprobte Signaturen: Das Beispiel der Tibetischen Pharmakologie und Pharmakotherapie}

Die Tibetische Medizin ist eine der großen überlieferten Medizintraditionen Asiens [19]. In der Schweiz begann 1969 mit Gründung der Firma Padma AG in Zürich die Herstellung Tibetischer Formeln unter modernen Qualitätsgesichtspunkten. 1970 wurde Padma Lax ${ }^{\circledR}$ und 1977 Padma $^{\circledR} 28$ in der Schweiz als Arzneimittel zugelassen [20]. Die dokumentierte Tibetische Tradition und die Daten aufgrund der westlichen regulatorischen Vorgaben ermöglichen eine vergleichende Betrachtung solcher Heilmittel.

Gemäß den Prinzipien der Tibetischen Medizin [21] werden Krankheiten primär in kalte und heiße Erkrankungen eingeteilt und als Verschiebung des individuellen Gleichgewichts der 3 Körperenergien («humors») rLung, Tripa und Beken - grob übersetzt als Wind, Galle und Schleim - verstanden. In der Therapie werden unter anderem Arzneiformeln eingesetzt, die üblicherweise aus 3-20 und mehr Komponenten bestehen. Meist handelt es sich um pflanzliche und zum Teil auch um mineralische Inhaltsstoffe.

Analog der Klassifizierung der Krankheiten teilt die traditionelle Pharmakologie [21] Arzneisubstanzen in «kühlende» und «wärmende» Stoffe ein aufgrund der beobachteten Wir- 


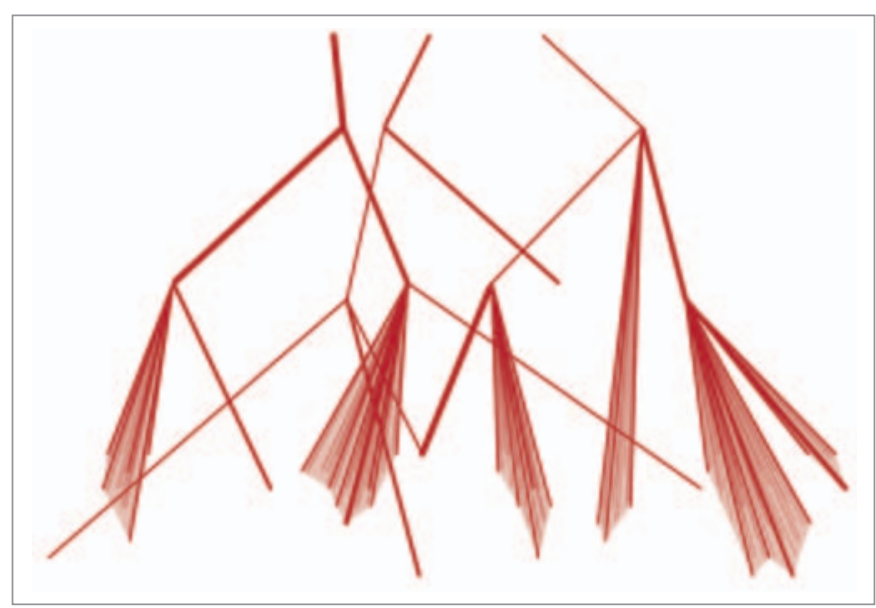

Abb. 3. Beispielhafte Komplexe Wirksignatur eines Phytotherapeutikums, die den pleiotropen Charakter und den Multi-Target-Wirkmechanismus über mehrere Ebenen des Organismus aufzeigt.

kungen auf die 3 Körperenergien. Die Eigenschaften der Arzneistoffe sind für den Tibetischen Arzt und Pharmakologen an sensorischen Eigenschaften wie Geschmack, Textur und Farbe erkennbar. Die getrockneten und pulverisierten, ansonsten jedoch unbehandelten Rohmaterialien werden so zu Formeln zusammengestellt, dass sie die krankheitsverursachenden Störungen der «Energiebalance» ausgleichen und den Organismus wieder ins Gleichgewicht zurückführen - so die traditionelle Theorie.

Überträgt man diesen empirisch erprobten Entstehungsweg in die systemische Sichtweise, erkennt man wieder die Entsprechung der Signatur Krankheit mit der Signatur Arzneimittel, in diesem Fall abgebildet auf das energetische Muster der Tibetischen Medizin.

Weitere Aspekte bei der Zusammenstellung einer Rezeptur [21] sind die erwarteten additiven, synergistischen und partiell antagonistischen Effekte; d.h., es werden Stoffe mit Hauptwirkung eingesetzt (etwa $80 \%$ einer Formel). Weiter werden Stoffe eingesetzt, die die Hauptwirkung unterstützen, sowie Substanzen, die eventuelle irritierende Wirkungen der anderen Inhaltsstoffe neutralisieren. Damit wirken sie nach einem Multi-Target-Mechanismus und erfüllen damit im Extrem die Eigenschaften der Pleiotropie, der Nicht-Spezifität und der Plastizität, die unter anderem für Rezepturen traditioneller asiatischer Medizinsysteme typisch sind.

Im Gegensatz zur regulatorisch-fixen europäischen bzw. westlichen Phytotherapie kann daher z.B. bei einer Tibetischen Vielstoffkombination ein Inhaltsstoff durch einen äquivalenten anderen ersetzt werden, ohne dass sich die Signatur der Formel ändert. Dieser Austausch von Komponenten bei gleichbleibendem Wirkprofil erlaubt es dem Tibetischen Arzt, auf Schwierigkeiten bei der Rohstoffversorgung sowie auf regionale botanische Gegebenheiten zu reagieren, ohne die Essenz der Formel zu ändern. Diese Praxis der asiatischen Medizintraditionen ist im gegenwärtigen europäischen wissenschaftlichen und regulatorischen Umfeld noch kaum verstanden und auch in den Pharmakopöen nicht entsprechend abgebildet. Die europäische bzw. westliche Fixierung auf botanische Spezies als einzig mögliche Art der Standardisierung (starre Kombinationen) schränkt das verfügbare Repertoire an Pflanzen zunehmend ein, während etwa die Tibetische Medizin mit großer Flexibilität auf verschiedene Herausforderungen reagieren kann.

\section{Netzwerk-Arzneien bei Multimorbidität}

Durch moderne Forschungsergebnisse $\mathrm{zu}$ traditionellen Arzneiformeln auf den verschiedenen Hierarchiestufen lässt sich auch im Netzwerk-Schema ein Wirkprofil bezeichnen. Im Fall der Vielkomponenten-Mischungen der Tibetischen Medizin ergibt sich ein ausgesprochen breit gefächertes, durch schwache Wechselwirkungen in den unteren Systemebenen abgestütztes Muster. Dies ist die pleiotrope Signatur des Arzneimittels (Abb. 1), das aufgrund seiner Vernetzungen zugleich als Netzwerk-Arznei charakterisiert werden kann.

Im Hinblick auf die Semiotik multimorbider Zustände scheinen Netzwerk-Arzneien, wie sie z.B. die pflanzlichen Vielstoffgemische der Tibetischen Medizin darstellen, besonders geeignet bei multimorbiden Patienten. Sie können im Rahmen einer Basistherapie in Kombination mit selektiven Arzneimitteln oder auch zur Behandlung spezifischer Symptome oder Symptomkomplexe eingesetzt werden.

Dabei ist natürlich eine Rezeptur zu wählen, deren Signatur eine zumindest teilweise Überlappung mit der Signatur des Krankheitsbildes aufweist. Als Beispiel mag die Symptomatik der peripheren Durchblutungsstörung dienen. Hierbei bezieht der Tibetische Arzt die Lebensstilfaktoren wie Ernährungsstatus, klimatische Bedingungen oder Aktivitätsmuster ebenso mit ein wie die Ergebnisse traditionell-subtiler Methoden wie Pulstasten oder Urindiagnose. Verallgemeinert kann im Rahmen der Tibetischen Medizin festgestellt werden, dass Patienten mit arteriosklerotischen Beschwerden ein Belastungsprofil haben, das die «Hitze» (Tripa) im Blutsystem erhöht und dort zugleich zu einer Reduktion des «beweglichen Anteils» (rLung) führt. Für das therapeutische Prinzip ergibt sich daraus eine Arznei, die «kühlend» ist und «anregend» auf die Blutzirkulation wirkt, was in der Rezeptur Padma 28 aufgegriffen ist [14, 22, 23]. Die moderne Signatur dieser Formel besteht unter anderem in den Eigenschaften anti-entzündlich, anti-oxidativ und durchblutungsfördernd. Es zeigt sich somit, dass im Prinzip eine relevante Kongruenz der beiden Erklärungsweisen möglich ist.

Durch den systemischen Ansatz gelingt es, traditionelle Arzneimittel als pleiotrope Signaturen zu verstehen, die im gesamten Netzwerk wirken. Der Transfer zwischen den verschiedenen Koordinatensystemen traditionell/modern/wissenschaftlich erscheint möglich und sinnvoll, wie am Beispiel der Rezeptur Padma 28 dargestellt wurde. Mit systemtheoretischen und netzwerkpharmakologischen Konzepten stehen wir am Anfang eines spannenden Forschungsansatzes, der empirisches Wissen mit moderner Medizin verbindet. Damit ist die systemische Betrachtungsweise auch ein Weg, die Anschlussfähigkeit traditioneller, komplementärmedizinischer Methoden an die anderen Disziplinen der modernen Medizin zu ermöglichen. Dies ist ein wesentlicher Aspekt für einen integrativen Ansatz.

\section{Disclosure Statement}

H.S. und C.V. sind Mitarbeiter der Padma AG. Ansonsten bestehen keine Interessenskonflikte. 


\section{Literatur}

1 Bopp M, Holzer BM: Prävalenz von Multimorbiditat in der Schweiz - Definitionen und Datenquellen. Praxis 2012;101:1609-1613.

2 Mercer SW, Gunn J, Bower P, Wyke S, Guthrie B: Managing patients with mental and physical multimorbidity. BMJ 2012;345:e5559.

3 Varela FJ: Principles of Biological Autonomy. New York, North Holland, 1979.

4 Luisi PL: Autopoiesis: a review and a reappraisal. Naturwissenschaften 2003;90:49-59.

$\checkmark 5$ Barabasi AL, Gulbahce N, Loscalzo J: Network medicine: a network-based approach to human disease. Nat Rev Genet 2011;12:56-68.

6 Simko GI, Gyurko D, Veres DV, Nanasi T, Csermely P: Network strategies to understand the aging process and help age-related drug design. Genome Med 2009;1:90.

7 del Sol A, Balling R, Hood L, Galas D: Diseases as network perturbations. Curr Opin Biotechnol 2010;21:566-571.

8 Ghazalpour A, Doss S, Yang X, et al.: Thematic review series: the pathogenesis of atherosclerosis. Toward a biological network for atherosclerosis. J Lipid Res 2004;45:1793-1805.

9 Vidal M, Cusick ME, Barabasi AL: Interactome networks and human disease. Cell 2011;144:986-998.
10 Csermely P, Agoston V, Pongor S: The efficiency of multi-target drugs: the network approach might help drug design. Trends Pharmacol Sci 2005;26: 178-182.

11 Saller R, Rostock M: Multimorbiditat und MultiTarget-Therapie in der Phytotherapie. Praxis 2012; 101:1637-1642.

12 Efferth T, Koch E: Complex interactions between phytochemicals. The multi-target therapeutic concept of phytotherapy. Curr Drug Targets 2011;12: 122-132.

13 Ma XH, Zheng CJ, Han LY, et al.: Synergistic therapeutic actions of herbal ingredients and their mechanisms from molecular interaction and network perspectives. Drug Discov Today 2009;14: 579-588.

14 Schwabl H, Vennos C: Der Multi-Target-Ansatz Tibetischer Heilmittel. Wirkmechanismen von Padma 28 im entzündlichen Geschehen am Beispiel der Arteriosklerose. Schweiz Z Ganzheitsmed 2006; 18:213-218.

15 Saller R, Holzer B: Multimorbiditat, Komorbiditat und phytotherapeutische Vielstoffgemische als Arzneimittel. Forsch Komplementmed 2010;17:300-302.

16 Saller R, Melzer J, Rostock M: Antiinflammatorisch wirksame Phytotherapeutika und ihr mögliches Potential bei tumorkranken Menschen. Forsch Komplementmed 2011;18:203-212.
17 Gertsch J: Botanical drugs, synergy, and network pharmacology: forth and back to intelligent mixtures. Planta Med 2011;77:1086-1098.

18 Mencher SK, Wang LG: Promiscuous drugs compared to selective drugs (promiscuity can be a virtue). BMC Clin Pharmacol 2005;5:3.

19 Kletter C, Kriechbaum M (eds): Encyclopedia of Tibetan Medicinal Plants. Stuttgart, Medpharm, 2001

20 Schwabl H, Geistlich S, McHugh E: Tibetische Arzneimittel in Europa: historische, praktische und regulatorische Aspekte. Forsch Komplementmed 2006;13(suppl 1):1-6.

21 Nikolayev S: Traditional principles of pharmacotherapy in Tibetan medicine; in Bazaron E (ed): Tibetan Medicine: The History, Methodology of Studies and Prospects of Use. Ulan-Ude, Buryat Scientifical Centre, The Biology Institute, 1998, pp 81-92.

22 Ueberall F, Fuchs D, Vennos C: Das anti-inflammatorische Potential von Padma 28 - Übersicht experimenteller Daten zur antiatherogenen Wirkung und Diskussion des Vielstoffkonzepts. Forsch Komplementmed 2006;13(suppl 1):7-12.

23 Saller R: Tibetische Kräutermischungen als moderne Medikamente. Komplexe mehrdimensionale Arzneimittelwirkungen als eine Komponente von Ganzheitlichkeit; in Reichle F (ed): Das Wissen vom Heilen. Aarau, AT-Verlag, 2012, pp 123-130. 